\title{
Corneal Shape, Volume, and Interocular Symmetry: Parameters to Optimize the Design of Biosynthetic Corneal Substitutes
}

\author{
Georges M. Durr, ${ }^{1}$ Edouard Auvinet ${ }^{2}$ Jeb Ong, ${ }^{1,3}$ Jean Meunier, ${ }^{1,2}$ and Isabelle Brunette ${ }^{1,4}$ \\ ${ }^{1}$ Department of Ophthalmology, University of Montreal, Montreal, Quebec, Canada \\ ${ }^{2}$ Department of Computer Science and Operations Research, University of Montreal, Montreal, Quebec, Canada \\ ${ }^{3}$ Department of Ophthalmology, McGill University, Montreal, Quebec, Canada \\ ${ }^{4}$ Maisonneuve-Rosemont Hospital Research Center, Montreal, Quebec, Canada
}

Correspondence: Isabelle Brunette, Department of Ophthalmology, CSARC-F117, Maisonneuve-Rosemont Hospital, 5415 boulevard de L'Assomption, Montreal, QC H1T 2M4 Canada;

i.brunett@videotron.ca.

Submitted: February 18, 2015

Accepted: April 11, 2015

Citation: Durr GM, Auvinet E, Ong J, Meunier J, Brunette I. Corneal shape, volume, and interocular symmetry: parameters to optimize the design of biosynthetic corneal substitutes. Invest Ophthalmol Vis Sci. 2015;56:4275-4282. DOI:10.1167/ iovs. $15-16710$
Purpose. To characterize the three-dimensional (3D) shape, volume distribution, and mirror symmetry of the right and left corneas at the scale of a large population, based on the integrated analysis of 3D corneal shape average maps and topography parameters.

Methods. A total of 7670 Orbscan II corneal topographies from 3835 consenting subjects with no history of ocular disease were studied. Average topography maps were created using the right and left corneal topographies of all subjects. To quantify symmetry, left eye topographies were flipped horizontally into "right eye" topographies and statistics maps were generated, including difference and intraclass correlation coefficient (ICC) maps.

RESults. The standard deviation of the anterior and posterior average elevation maps in the 3$\mathrm{mm}$ radius central zone of the right and left corneas ranged within $\pm 8 \mu \mathrm{m}$ and $\pm 44 \mu \mathrm{m}$, respectively. The ICC maps showed almost perfect interocular agreement for anterior elevation, posterior elevation, and pachymetry (all ICCs $>0.96$ ). All studied shape parameters also showed excellent agreement (ICCs $\geq 0.80$ ). Mirror symmetry was not affected by age, sex, or spherical equivalent. We also showed that this horizontal reflection (flip) of the right and left corneal shapes could not be replaced by a simple rotation.

Conclusions. These results indicate that in normal eyes, the anterior elevation, posterior elevation, and pachymetry of the right and left corneas show remarkable symmetry. This comprehensive analysis was achieved with the purpose of guiding the development of future biosynthetic corneal substitutes.

Keywords: corneal topography, interocular symmetry, enantiomorphism, artificial cornea, corneal transplantation
$\mathrm{C}$ orneal surgery and corneal replacement techniques have witnessed profound changes during the past decades, with the successive introduction of excimer laser surgery, femtosecond laser surgery, and the change in paradigm from fullthickness corneal replacement (penetrating keratoplasty) to selective lamellar replacement of the diseased tissue (anterior or posterior lamellar keratoplasty). In line with this evolution, the artificial cornea holds the promise of a major evolution in the concept of corneal replacement.

Fagerholm et al. ${ }^{1}$ recently published the 4-year follow-up results of a phase I safety study in which biosynthetic analogues of human corneal stromal matrices were implanted in the first 10 human patients. These biosynthetic stromal implants remained stably integrated and, by mimicking the extracellular matrix of the cornea, promoted host tissue regeneration and repopulation by host epithelial cells, nerves, and keratocytes. A number of biomaterials are being studied for the production of corneal substitutes so as to replace a diseased cornea without the need for human eye bank tissue. ${ }^{2-4}$

Biosynthetic corneal substitutes offer significant advantages over eye bank native transplants, including sterility, absence of risk of immune rejection, quality control, and the possibility of high-volume production. One of the unique advantages of corneal substitutes over native tissue is the possibility of tailoring their shape, aiming at a more successful postoperative outcome. Corneal shape is of primary importance, as it determines most of the eye's total refractive power and, hence, visual acuity.5,6 The first implants described by Fagerholm et al. ${ }^{1}$ were produced with contact lens molds and used for partial-thickness corneal replacement, using an anterior lamellar keratoplasty surgical technique. Additional data are thus needed to guide the geometry of full-thickness biosynthetic corneal stromal substitutes.

In this context, a thorough understanding of the corneal shape at a population level becomes essential, and to the best of our knowledge, there are no comprehensive studies on the global three-dimensional (3D) shape of the normal human cornea that brings sufficient information to guide the conception and production of biosynthetic corneal substitutes. A better knowledge of the variation in size and volume distribution of normal corneas will allow optimizing the shape of the implants. A better awareness of the concepts of intracorneal asymmetry and interocular symmetry will also allow establishing guidelines regarding laterality and orienta- 
tion of these implants. For instance, it will allow deciding if there should be implants for right eyes and implants for left eyes. It will also allow deciding if an implant can be rotated freely within its recipient bed (as is currently done during corneal transplantation using eye bank tissue) or if its orientation should be respected.

With this in mind, the goal of this study was to characterize and quantify the 3D shape and mirror symmetry of the right and left corneas, based on the integrated analysis of 3D corneal shape average maps and topography parameters in a large series of healthy subjects.

\section{Materials ANd Methods}

\section{Study Subjects and Topography System}

The research protocol of this cross-sectional study was approved by the Maisonneuve-Rosemont Hospital human experimentation committee (Montreal, QC, Canada) and all patients signed a research consent form. The Quebec Corneal Shape and Function Database (Montreal, QC, Canada), which compiles more than 36,000 corneal topographies, was screened according to the following inclusion criteria: no history of ocular disease, ocular surgery, or recent contact lens wear, and age and refraction data available. Corneal topographies of both eyes were recorded on the same day. A calibrated Orbscan topographer (Model IIz, Version 3.12; Bausch and Lomb, Rochester, NY, USA) was used.

\section{Average Maps Construction}

This study was based on the construction and analysis of a 3Dshape corneal atlas. A topographical atlas typically includes a series of maps characterizing corneal shape at the scale of a population. Average anterior elevation maps, average posterior elevation maps, and average pachymetry maps were generated for the right (OD) and left (OS) eyes. Statistics maps, including difference maps and intraclass correlation coefficient (ICC) maps, also were generated, and the data were completed using focal corneal shape parameters.

The atlas construction methodology used in this study has in part been described elsewhere. ${ }^{7}$ Briefly, average corneal topography maps were produced from individual topography maps. Before averaging, the anterior surface best fit sphere (BFS) of each cornea was calculated and the mean anterior BFS of all right and left corneas was used as a reference. The BFS represents the sphere that best adjusts to the corneal surface with least-squares regression, in a central adjustment zone of $10.0 \mathrm{~mm}$ in diameter. Each cornea was then spatially normalized by aligning their respective anterior BFS on the mean anterior BFS with isometric scaling and translation. This normalization step was necessary to register corneas of different subjects into the same reference coordinate system and consequently to remove the effect of their various (global) sizes and various 3D positions in space while preserving each cornea local shape features. Note that similar normalization procedures are used for other medical imaging modalities (e.g., brain imaging). Each cornea was treated as a volume, by always applying the same normalization step to the anterior and posterior surfaces together. The elevation topographies were then averaged. The pachymetry was computed along the radius emerging from the center of the mean anterior BFS between the posterior and anterior normalized surfaces. Average maps were generated for the right and for the left corneas.

An average map has the same appearance as an individual map, the only difference being that each color point on the average map represents the average at this specific point of all the individual measurements for the studied population. To facilitate interpretation, the color scales used for the average maps were the same as those commonly used in the commercial topography system for individual topographies $(5-\mu \mathrm{m}$ color steps for the elevation maps, green representing a point on the BFS, and $20-\mu \mathrm{m}$ color steps for the pachymetry maps).

To assess interocular symmetry in shape of the right and left corneas, a mirrored image of each left eye topography was generated by flipping the left eye topography horizontally across the vertical meridian. This mirrored image of the left eye (OShf) topography was then subtracted from its mate OD topography. Difference maps were computed so that each color point in a difference map represented the average of all paired differences between OD and OShf at this specific point. An ICC map complemented the difference map to assess the agreement between the right and left corneal topographies.

We then tried to assess to which extent rotation of an implant that has the typical shape of a left cornea could allow to approximate the shape of a typical right cornea. For this, counter-clockwise rotations over 360 degrees were imposed to the left eye topography before subtracting it from its mate OD topography; OD-OS difference maps were then computed for each rotation step. To quantify the amount of mismatch illustrated by these difference maps, the root mean square (RMS) difference was calculated for each difference map. A higher RMS value represents a greater difference in shape between the right and rotated left topographies.

\section{Shape Parameters}

Several shape parameters commonly used in corneal topography also were studied to complement the information brought by the average maps.

A series of corneal shape parameters generated by the Orbscan software were studied. Their definitions are listed in Table 1 . In the central $1.5-\mathrm{mm}$ radius central zone, astigmatism, maximum and minimum keratometry readings, and their corresponding meridian were examined. In the 3 -mm radius central zone, as well as in the 3- to 5-mm ring-shaped paracentral zone, mean power, astigmatism power, steep and flat axes, and irregular astigmatism were evaluated. Pachymetry of the thinnest point on the cornea and the white-to-white horizontal diameter also were analyzed.

A series of shape parameters also were calculated from the individual topographies, including the BFS radius, asphericity, apical radius of curvature, $(x, y, z)$ coordinate of the anterior and posterior surfaces apices, as well as the corneal volume in the $1.5-$ and $3.0-\mathrm{mm}$ radius central zones. Asphericity $(Q)$ indicates the rate at which the corneal curvature changes from the center to the periphery. In a perfect sphere, $Q=0$. A $Q$ value less than 0 indicates that the corneal surface curvature gradually flattens from center to periphery (prolate shape). A $Q$ value greater than 0 indicates that the corneal curvature gradually steepens from center to periphery (oblate). The apical radius of curvature $(R)$ characterizes the circle tangent to the apex (point of greatest curvature). The smaller the $R$ value, the greater is the curvature, and vice versa. The $R$ and $Q$ values were obtained by leastsquares, fitting the following equation to the $5-\mathrm{mm}$ radius central region of each elevation map ${ }^{8}$.

$$
x^{2}+y^{2}+(1+Q) z^{2}-2 R z=0
$$

\section{Statistical Analysis}

Mean values and SDs are reported. For angular data (in degrees), circular statistics were used to compute mean and SD values. ${ }^{9}$ The ICC was used to assess agreement between right 
TABLE 1. Topography Parameters, With Their Abbreviations, Units, and Definitions

\begin{tabular}{llll}
\hline \multicolumn{1}{c}{ Parameter } & \multicolumn{1}{c}{ Abbreviation } & Unit & Definition \\
\hline Astigmatism & Astig & $\mathrm{KD}$ & Astigmatism in the 1.5-mm radius central zone \\
Maximum keratometry & Max K & $\mathrm{KD}$ & Maximal curvature in the 1.5-mm radius central zone \\
Minimum keratometry & Min K & $\mathrm{KD}$ & Minimal curvature in a 1.5-mm radius central zone \\
Mean power & $0-3$ and 3-5 Mean Pwr & $\mathrm{KD}$ & Average keratometric power in specified zone \\
Astigmatic power & $0-3$ and 3-5 Astig Pwr & $\mathrm{KD}$ & Astigmatism in specified zone \\
Steep axis & $0-3$ and 3-5 Steep Axs & $\mathrm{deg}$ & Axis of maximum curvature in specified zone \\
Flat axis & $0-3$ and 3-5 Flat Axs & $\mathrm{deg}$ & Axis of the minimum curvature in specified zone \\
Irregularity & $0-3$ and 3-5 Irreg & $\mu \mathrm{m}$ & Irregular astigmatism index \\
Minimal pachymetry & Pac Min & $\mathrm{mm}$ & Thinnest point on the cornea \\
Horizontal white-to-white & HWTW & Horizontal corneal diameter
\end{tabular}

Pwr, power; KD, keratometric diopter; deg, degree.

and left corneas. An ICC of 0 to 0.2 was considered as poor; 0.3 to 0.4 , fair; 0.5 to 0.6 , moderate; 0.7 to 0.8 , strong; and greater than 0.8 was considered as almost perfect. A multiple linear regression model including interaction was used to investigate the effect of age, sex, and refraction on the linear relationship between the right and left corneas. The Studentized residuals and Cook's distance were calculated to detect outliers and observations with high influence. The analyses were conducted using SAS 9.2 (SAS Institute, Inc., Cary, NC, USA) for the shape parameters and Matlab R2012a (MathWorks, Natick, MA, USA) for average maps comparisons.

\section{Results}

\section{Population}

A total of 7670 eyes from 3835 subjects, 1874 females and 1961 males, were included in this study. The mean ( \pm SD) age was $39.0 \pm 10.3$ years (range, 5.8-100.0 years). The mean refractive error was $-3.57 \pm 2.25$ diopters (D) (range, -12.00 $4.50 \mathrm{D}$ ) for the right eyes and $-3.58 \pm 2.26 \mathrm{D}$ (range, $-15.25-$ $4.38 \mathrm{D})$ for the left eyes. The refractive spherical equivalents of the right and left eyes showed almost perfect agreement (ICC $=$ 0.97).

\section{Average Corneal Topography Maps}

Anterior Elevation Maps. The anterior elevation maps of both eyes showed a typical concentric pattern, in which the apex in warm colors (i.e., above the BFS) was surrounded by cold colors (under the BFS), themselves surrounded by a ring of increasingly warmer colors toward the periphery (Figs. 1A, 1B). A slightly elevated isthmus joined the central cornea to the temporal periphery. In the right eye, the apex laid $9 \mu \mathrm{m}$ above its BFS and was slightly displaced in the inferior temporal direction $(-0.412 \mathrm{~mm},-0.275 \mathrm{~mm})(x, y)$. In the left eye, the apex laid $10 \mu \mathrm{m}$ above the BFS and was also slightly displaced in the inferior temporal direction $(0.686 \mathrm{~mm},-0.344 \mathrm{~mm})$. In both eyes, the SD of the anterior elevation was less than \pm 8 $\mu \mathrm{m}$ in the 3-mm radius central zone.

Posterior Elevation Maps. The posterior elevation maps of both eyes showed a concentric pattern similar to that of the anterior surface, with an isthmus joining the central cornea to the temporal periphery (Figs. 1F, 1G). In both eyes, the posterior apex laid $32 \mu \mathrm{m}$ above the BFS. This higher apex-toBFS distance of the posterior surface was in agreement with a mathematical model showing that the higher posterior apex elevation is due to the combination of a steeper apical curvature $(R)$ and increased prolateness (negative asphericity $Q)$ compared with the anterior corneal surface, as seen in Table $2 .{ }^{10,11}$ In both eyes, the posterior apex was slightly displaced in the inferior temporal direction with respect to the center of the map (OD: $-0.476 \mathrm{~mm},-0.298 \mathrm{~mm}$; OS: $0.333 \mathrm{~mm},-0.183$ $\mathrm{mm})$. The SD of the posterior elevation ranged within $\pm 44 \mu \mathrm{m}$ in the 3 -mm radius central zone.

Pachymetry Maps. Both right and left eyes average pachymetry maps showed a concentric pattern, thinner in the center and progressively thicker toward the periphery (Figs. 1K, 1L). In both eyes, the thinnest point measured $538 \pm$ $35 \mu \mathrm{m}$ (Table 2).

Difference Maps. The difference maps showed that the differences in anterior elevation between OD and OShf ranged within $\pm 6 \mu \mathrm{m}$, as illustrated by the almost entirely green difference map shown in Fig. 1D. Similarly, both the posterior elevation and pachymetry difference maps showed differences between OD and OShf within $\pm 16 \mu \mathrm{m}$ (Figs. 1I, 1N).

Intraclass Correlation Maps. The ICC values assessing interocular agreement for the anterior and posterior surfaces elevation (Figs. 1E, 1J; all ICC values $>0.99$ ) and pachymetry (Fig. 1O; all ICCs > 0.96) showed almost perfect agreement.

\section{Shape Parameters}

The mean values of the different corneal shape parameters for the right and horizontally flipped left eyes, differences between eyes, ICC, and 95\% confidence interval are listed in Table 2 and illustrated in Figures $2 \mathrm{~A}$ through $2 \mathrm{H}$. All of the shape parameters showed almost perfect agreement, with ICCs varying from 0.80 to 0.98 (Table 2).

The Studentized residuals and Cook's distance were calculated to check for possible outliers. A few observations had a Studentized residual greater than +2 or less than -2 and a large Cook's distance. These observations did not influence the estimates of the predictor equation and thus were not considered outliers.

\section{Effect of Age, Sex, and Refraction}

Age, sex, and spherical equivalent and their interaction with right corneas were added in turn to the linear regression model to determine how they might influence the linear relationship between the right and left corneas. The analysis was repeated for three topography parameters, namely Mean power in the 3$\mathrm{mm}$ radius central zone (Fig. $2 \mathrm{~A}$ ), Astigmatic power in the 0 - to 3-mm radius central zone (Fig. 2E), and Minimum pachymetry (Fig. 2G). None of the interactions were significant at a 5\% level, meaning that the linear relationship between the right and left corneas was not influenced by age, sex, and spherical equivalent. In other words, for the three topography parameters, mirror symmetry between the right and left corneas was not affected by age, sex, or spherical equivalent. 


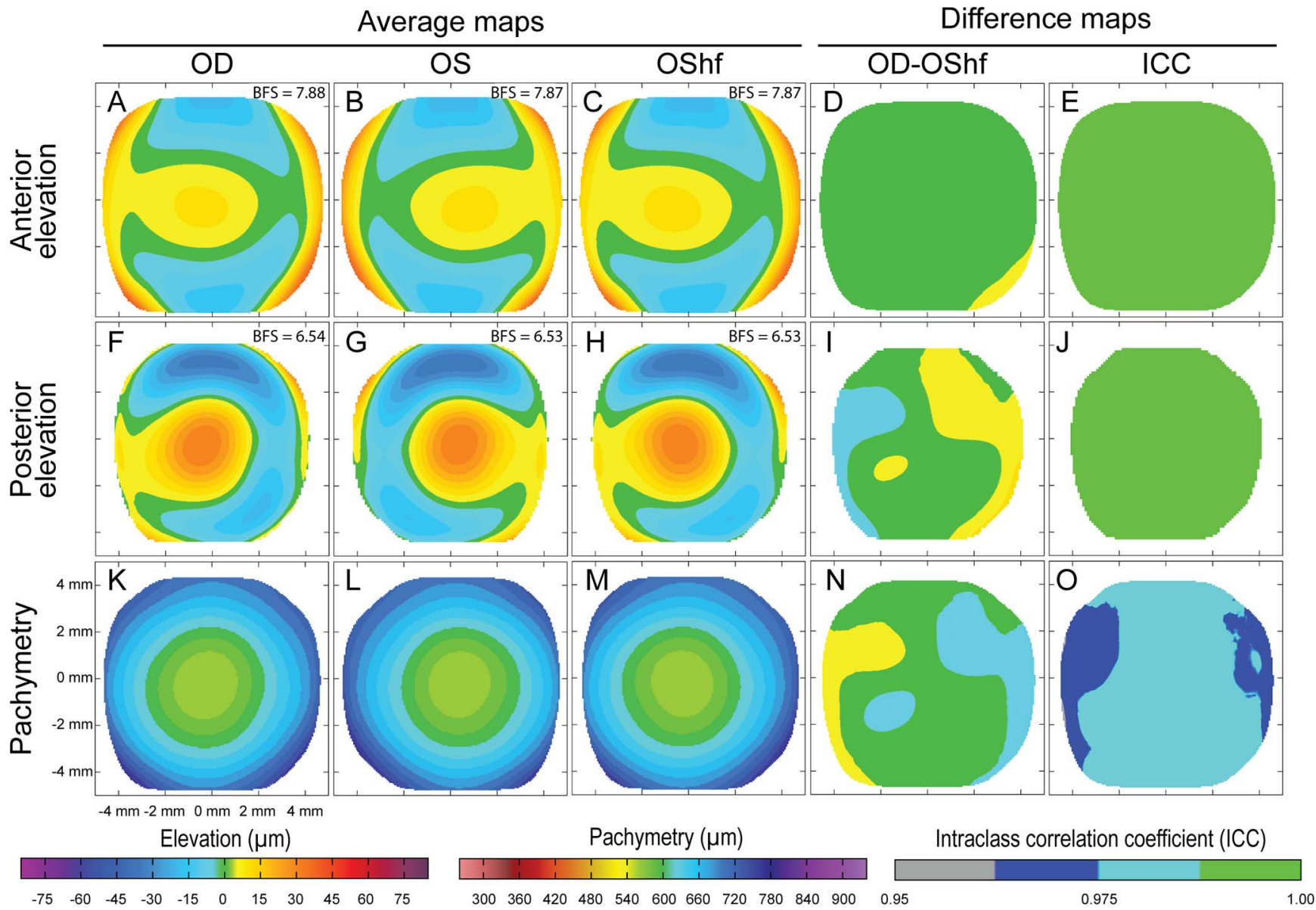

Figure 1. Three-dimensional corneal shape atlas. (A-E) Anterior elevation maps. (F-J) Posterior elevation maps. (K-O) Corneal pachymetry maps. The first column illustrates the right eye maps $(\mathbf{A}, \mathbf{F}, \mathbf{K})$; the second column, the left eye maps $(\mathbf{B}, \mathbf{G}, \mathbf{L})$; the third column, the horizontally flipped left eye maps $(\mathbf{C}, \mathbf{H}, \mathbf{M})$; the fourth column illustrates difference maps $(\mathbf{D}, \mathbf{I}, \mathbf{N})$; and the fifth column, ICC maps $(\mathbf{E}, \mathbf{J}, \mathbf{O})$. Difference maps were computed so that each color point in a difference map represented the average of all paired differences between OD and OShf at this specific point. An ICC map complemented each difference map to assess the agreement between the right and left corneal topographies.

\section{Effect of Rotation}

To assess to which extent an implant that has the typical shape of a left cornea could be rotated to mimic the typical shape of a right cornea, stepwise rotations were imposed to the left eye topography before subtracting it from its mate OD topography. Supplementary Figure S1 illustrates the effect of such rotation on the anterior elevation difference map. The OD-OS difference maps are represented as a function of degrees of rotation of OS map (only 45-degree steps are illustrated here). Similarly, Supplementary Figure S2 illustrates the effect of rotation on the pachymetry difference map. In both cases, one can notice that none of the rotations has allowed a better fit than that of a simple horizontal flip of the left eye topography across the vertical meridian, which is referenced in the center of the figure.

To quantify the amount of mismatch illustrated by these difference maps, the RMS difference was calculated for each difference map (Fig. 3). Column 1 gives the RMS value of the OD-OS difference for the anterior elevation (Fig. 3A), posterior elevation (Fig. 3C), and pachymetry (Fig. 3E) maps. It shows that the closest match (lowest RMS value) between the anterior surfaces of the right and left eyes was obtained by rotating the left cornea by 180 degrees (Fig. 3A; white arrow). This amount of rotation, however, did not optimally reduce the mismatch between the posterior surface and pachymetry maps of the two eyes, which instead were improved by rotations of 225 and 270 degrees, respectively (Figs. 3C, 3E; white arrows). Column 2 shows that the best fit between the right and left corneas was obtained by flipping the left eye topography horizontally without further rotation (Figs. 3B, 3D, 3F; black arrows).

\section{Discussion}

To the best of our knowledge, this is the first in-depth report on corneal shape and symmetry of the right and left corneas at the scale of a population, based on the integrated analysis of 3D shape average maps and topography parameters. The analysis of a large series of 7670 normal eyes showed that the 3D shapes of the right and left corneas show remarkable symmetry. The anterior elevation, posterior elevation, and pachymetry maps of the right corneas mirrored those of the left corneas, and the 3D shape parameter values for both eyes showed almost perfect agreement. We also showed that this horizontal reflection (flip) of the right and left corneal shapes could not be replaced by a simple rotation.

\section{Advantages of the Atlas Methodology}

The average model or atlas methodology is a powerful tool for detailed characterization of the regional distribution of corneal shape and volumes. Average maps can be produced using any 
TABLE 2. Refraction and Corneal Shape Data for Right and Horizontally Flipped Left Eyes, Absolute Differences Between Eyes, ICC, and 95\% Confidence Interval

\begin{tabular}{|c|c|c|c|c|c|}
\hline & OD, Mean \pm SD & OShf, Mean \pm SD & $\begin{array}{l}\text { Difference } \\
\text { OD - OShf }\end{array}$ & ICC & $95 \% \mathrm{CI}$ \\
\hline \multicolumn{6}{|l|}{ Refraction } \\
\hline Spherical equivalent, D & $-3.57 \pm 2.25$ & $-3.58 \pm 2.26$ & 0.02 & 0.973 & $0.971-0.975$ \\
\hline Cylinder, D & $0.72 \pm 0.74$ & $0.76 \pm 0.76$ & 0.04 & 0.876 & $0.868-0.884$ \\
\hline Axis, degree & $89 \pm 35$ & $90 \pm 34$ & 1.00 & 0.934 & $0.929-0.939$ \\
\hline \multicolumn{6}{|l|}{ Anterior elevation maps } \\
\hline $\mathrm{BFS}$ radius, $\mathrm{mm}$ & $7.88 \pm 0.24$ & $7.87 \pm 0.24$ & 0.01 & 0.985 & $0.983-0.987$ \\
\hline Asphericity, $Q$ & $-0.26 \pm 0.12$ & $-0.25 \pm 0.12$ & 0.01 & 0.853 & $0.843-0.863$ \\
\hline Radius of curvature, $R$ & $7.65 \pm 0.26$ & $7.64 \pm 0.27$ & 0.01 & 0.973 & $0.971-0.975$ \\
\hline \multicolumn{6}{|l|}{ Posterior elevation maps } \\
\hline $\mathrm{BFS}$ radius, $\mathrm{mm}$ & $6.55 \pm 0.25$ & $6.53 \pm 0.25$ & 0.01 & 0.984 & $0.984-0.986$ \\
\hline Asphericity, $Q$ & $-0.58 \pm 0.22$ & $-0.57 \pm 0.22$ & 0.02 & 0.873 & $0.864-0.881$ \\
\hline Radius of curvature, $R$ & $6.03 \pm 0.27$ & $6.03 \pm 0.27$ & $<0.01$ & 0.940 & $0.936-0.944$ \\
\hline \multicolumn{6}{|l|}{ Volume of corneal zones } \\
\hline $1.5-\mathrm{mm}$ radius central, $\mathrm{mm}^{3}$ & $4.32 \pm 0.27$ & $4.32 \pm 0.27$ & $<0.01$ & 0.988 & $0.987-0.989$ \\
\hline $3.0-\mathrm{mm}$ radius central, $\mathrm{mm}^{3}$ & $18.87 \pm 1.10$ & $18.88 \pm 1.11$ & 0.01 & 0.990 & $0.989-0.991$ \\
\hline \multicolumn{6}{|l|}{ Shape parameters } \\
\hline $\operatorname{Max} \mathrm{K}, \mathrm{KD}$ & $44.31 \pm 1.53$ & $44.38 \pm 1.54$ & 0.07 & 0.972 & $0.970-0.974$ \\
\hline Max M, deg & $272 \pm 26$ & $268 \pm 26$ & 4.00 & 0.873 & $0.862-0.883$ \\
\hline $\operatorname{Min} \mathrm{K}, \mathrm{KD}$ & $43.31 \pm 1.45$ & $43.35 \pm 1.46$ & 0.03 & 0.973 & $0.972-0.975$ \\
\hline Min M, deg & $182 \pm 26$ & $178 \pm 26$ & 4.00 & 0.972 & $0.970-0.975$ \\
\hline Pac min, $\mu \mathrm{m}$ & $538 \pm 35$ & $538 \pm 35$ & 0.28 & 0.984 & $0.983-0.985$ \\
\hline \multicolumn{6}{|l|}{ 0-3-mm zone } \\
\hline Mean pwr, KD & $43.79 \pm 1.44$ & $43.85 \pm 1.45$ & 0.06 & 0.980 & $0.978-0.981$ \\
\hline Astig pwr, KD & $1.00 \pm 0.66$ & $1.04 \pm 0.68$ & 0.04 & 0.862 & $0.853-0.871$ \\
\hline Steep axis, deg & $90 \pm 27$ & $90 \pm 26$ & 0.30 & 0.874 & $0.866-0.882$ \\
\hline Flat axis, deg & $180 \pm 26$ & $179 \pm 26$ & 1.00 & 0.972 & $0.970-0.974$ \\
\hline \multicolumn{6}{|l|}{ 3-5-mm zone } \\
\hline Mean pwr, KD & $43.51 \pm 1.41$ & $43.57 \pm 1.43$ & 0.06 & 0.983 & $0.981-0.984$ \\
\hline Astig pwr, KD & $1.07 \pm 0.70$ & $1.12 \pm 0.73$ & 0.05 & 0.798 & $0.784-0.811$ \\
\hline Steep axis, deg & $95 \pm 25$ & $94 \pm 24$ & 1.00 & 0.852 & $0.843-0.862$ \\
\hline Flat axis, deg & $182 \pm 25$ & $181 \pm 24$ & 1.00 & 0.976 & $0.974-0.977$ \\
\hline
\end{tabular}

CI, confidence interval.

type of topography system, as long as the raw data are available. In this study, elevation maps were preferred to curvature maps because they provide the true (absolute) topography needed to assess the full corneal shape (anterior and posterior surfaces and the associated pachymetry). However, any shape parameter could be analyzed and mapped (e.g., axial curvature, tangential curvature). Average elevation maps also allow easy illustration and comparison of the astigmatism axes without special management for circular data. Statistic maps allow regional quantification of the distribution, order of magnitude, and statistical significance of differences. The choice of the statistic is determined according to the need. In the present study, ICCs were preferred to Pearson correlation coefficients for the comparison of right and left eyes, as ICCs allow assessment of the degree of agreement between two measures, whereas the Pearson correlation coefficient only assesses linear relationships.

\section{Interocular Symmetry or Enantiomorphism}

Enantiomorphism is a common finding in the human body (ears, hands, feet). Overall symmetry of the right and left corneas, however, has only been sporadically studied, using different methodologies. Boote et al. ${ }^{12}$ mapped in detail the distribution and orientation of the collagen fibrils across the corneas of a pair of mate eye bank eyes using wide-angle $\mathrm{x}$-ray scattering. Although structurally distinct, the right and left corneas showed midline symmetry. Smolek et al., ${ }^{13}$ Wang et al., ${ }^{14}$ and Lombardo et al. ${ }^{15}$ observed a moderate to high degree of mirror symmetry between the higher-order aberrations of the right and left corneas, with significant intersubject variability. Corneal optical aberrations are known to be closely linked to corneal shape. ${ }^{16}$

A few other groups have studied symmetry between right and left corneas based on keratometry and/or corneal topography parameters, using subjective or objective and qualitative or quantitative methodologies. ${ }^{17-22}$ Although we could not find other studies similar to ours, our findings are in accordance with these reports. Dingeldein and Klyce ${ }^{18}$ subjectively observed a high degree of mirror symmetry in shape between the right and left corneas, based on the first corneal topography analyses in 1989 (LSU Eye Center corneal analysis system; Louisiana State University, New Orleans, LA, USA). A few studies subsequently reported quantitative comparisons of right and left eye corneal topographies, using the Orbscan ${ }^{17,20,22}$ or the Pentacam system (Oculus, Inc., 

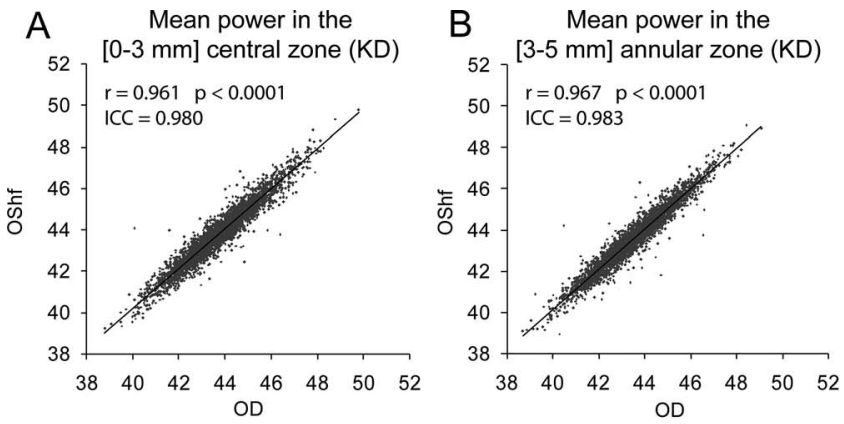

C Maximum keratometry (KD)
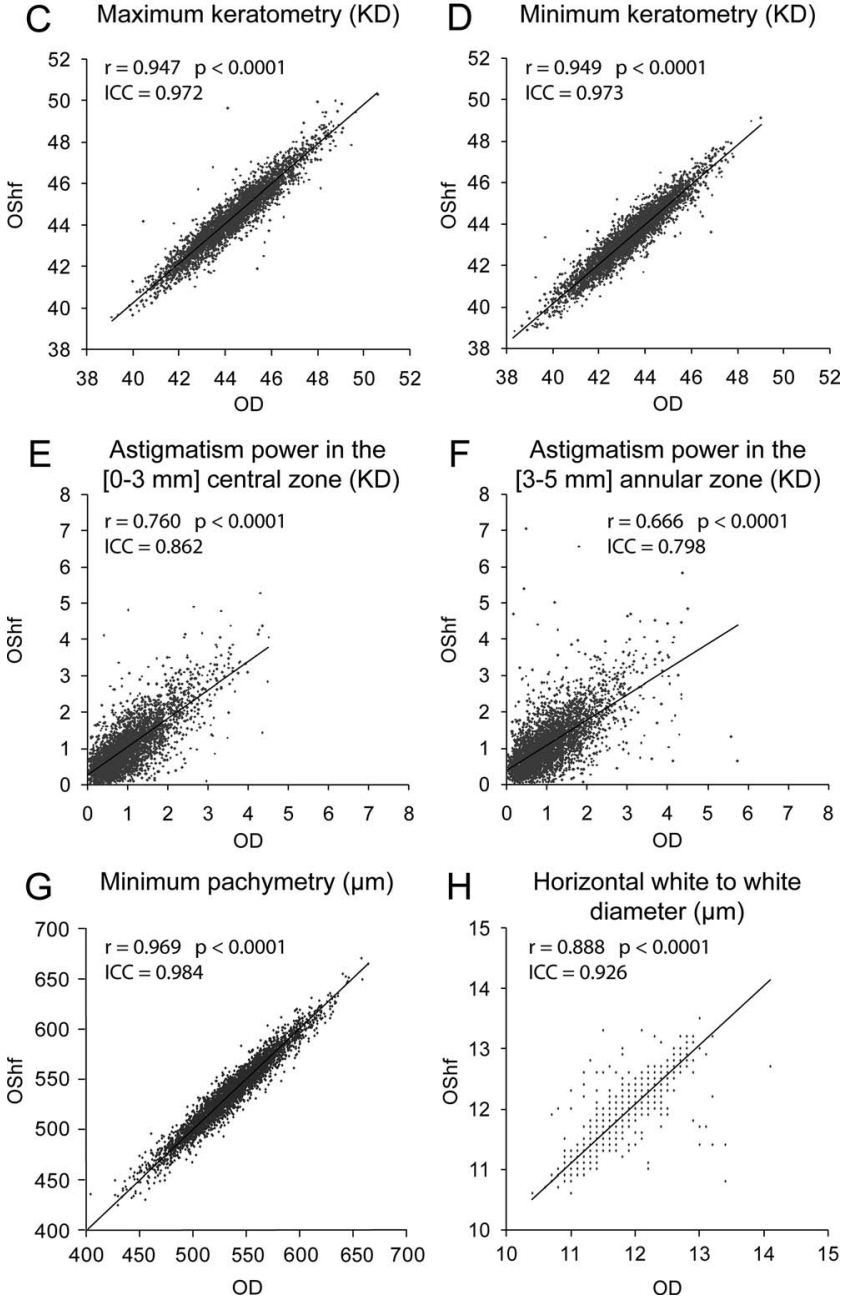

Figure 2. Distribution of the shape parameters of the right and horizontally flipped left corneas. Several shape parameters commonly used in corneal topography were also measured in the right and horizontally flipped left corneas and compared to complement the information brought by the average maps: (A) Mean power in the 3-mm radius central zone. (B) Mean power in the 3- to 5-mm ring-shape paracentral zone. (C) Maximum keratometry. (D) Minimum keratometry. (E) Astigmatic power in the 0- to 3-mm radius central zone. (F) Astigmatic power in the 3- to 5-mm paracentral zone. (G) Minimum pachymetry. (H) Horizontal white-to-white diameter. Linear regression lines, Pearson correlation coefficients, corresponding $P$ values, and ICC are shown.

Arlington, WA, USA). ${ }^{19}$ With variable methodologies and relatively small sample sizes ( 50 to 275 subjects), these groups found evidence of corneal symmetry based on small differences between eyes, with or without statistical analyses, and sometimes using correlations to assess proportionality between eyes. The studied parameters varied, including anterior and posterior surface shape parameters, such as simulated keratometry, steepest keratometry, central radius of curvature, apex elevation, and BFS radius, corneal volumes within central zones of given diameters, as well as central corneal thicknesses. ${ }^{17,19,20,22}$

A few other studies used a decrease in interocular symmetry as a useful tool to detect keratoconus. ${ }^{23-26}$ Although of significant interest in the context of preoperative screening before refractive surgery, this type of assessment based on specific topographical parameters is not meant to guide the production of corneal substitutes, as it informs only a little about global corneal shape, volume, and symmetry.

\section{Clinical Significance}

The differences observed in this study between the right and horizontally flipped left corneas were extremely small (Table 2) and their order of magnitude was clinically negligible. Regardless of the type of device used, the limits of this device also need to be taken into account. For instance, knowing that the repeatability of the Orbscan system, in ideal conditions, is in the order of 2 to $5 \mu \mathrm{m}$ in the center for anterior elevation measurements and decreases toward the periphery, ${ }^{27-29}$ the $0.28-\mu \mathrm{m}$ mean interocular difference in minimum pachymetry values observed in the present study clearly becomes irrelevant.

\section{Translation to Corneal Substitute Technology}

The new science of biosynthetic corneal substitutes is still in its earliest stages of development and, as mentioned, one of their unique and most promising advantages over human corneal tissue is the possibility of tailoring their shape. All the elements of the implant's shape can be controlled, including its anterior and posterior surfaces' shape, thickness, diameter, and orientation, all of which with the same goal of optimizing corneal rehabilitation and minimizing postoperative astigmatism. The comprehensive analysis of corneal shape and symmetry reported herein was achieved with the purpose of guiding the development of future biosynthetic corneal substitutes.

Although corneal surgeons have reached a plateau in terms of optimizing results with traditional corneal transplantation techniques, ${ }^{30-34}$ biomaterials technology offers an entirely new window of opportunity. The implant's shape also could be customized, taking into account the other optical elements of the eye, as it is done for the calculation of the intraocular lens power before cataract surgery. This would help reduce the amount of postoperative ametropia and would ensure greater precision and predictability. In case of unilateral disease, the mirror shape of the healthy contralateral cornea could be reproduced to generate the implant for the diseased cornea. This would help to prevent anisometropia (difference in refractive error between both eyes) and secondary aniseikonia (difference in the perceived size of images), which are known to lead to diplopia, disorientation, and headaches. Additional work will be needed to refine this model, based on the functional results obtained. Different imaging technologies also may be needed to complement the analysis.

\section{Laterality and Orientation}

Our results suggest that in a surgical context, a corneal implant having the shape of a right cornea should be implanted in the right eye, and a corneal implant having the shape of a left cornea should be implanted in the left eye. Our results also 

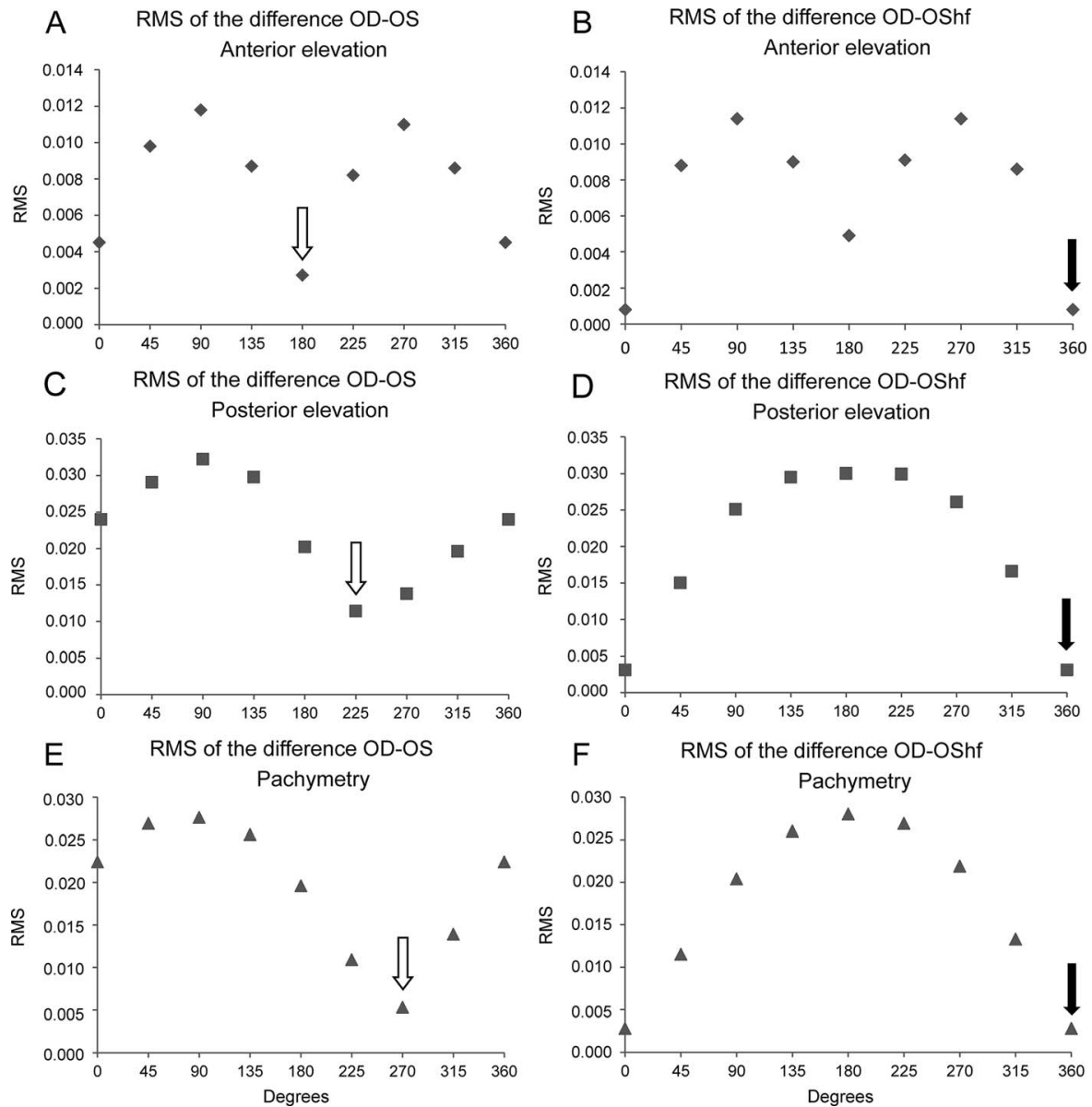

FiguRE 3. Root mean square difference between the right and left eye topographies as a function of rotation of the left eye topography. To quantify the amount of mismatch illustrated by the difference maps shown in Supplementary Figures S1 and S2, the RMS difference was calculated for each difference map: first column, OD-OS; second column, OD-OShf (i.e., with prior horizontal flip of the left eye topography across the vertical meridian). The same exercise was repeated for anterior elevation (first row), posterior elevation (second row), and pachymetry (third row). Rotations by 45-degree steps are illustrated here. A higher RMS value represents a greater difference in shape between the right and rotated left topographies. Arrows indicate the best fit between the two eyes resulting in minimal OD-OS difference (white arrow) or OD-OShf (black arrow).

indicate that rotation of a left eye implant would not make it similar to a right eye implant. These implants would not be interchangeable and their orientation should be respected.

The problem of severe postoperative astigmatism after traditional full-thickness corneal transplantation using human donor tissue has been very well documented. However, although abundant literature has been published exploring ways of reducing posttransplantation astigmatism by adapting the suturing technique (interrupted versus continuous running sutures), adjusting suture tension, and increasing the precision of the cut (manual versus mechanical versus laser trephination), ${ }^{35}$ only a few studies have examined the effect of graft positioning within the recipient bed, with variable results. ${ }^{36-38}$ Grütters et al. ${ }^{38}$ showed that the postoperative axis of the recipient's astigmatism is directly influenced by the orientation of the donor button. Implant's orientation may be even more influential, especially knowing that suture related astigmatism could be reduced by using larger implants without the risk for rejection inherent to eye bank allografts (immunologically inert biomaterials).

We think that this study responds to a significant need in that future refinement in the next generation of implant design will necessarily involve thorough knowledge of corneal shape and symmetry so as to improve postoperative shape control and predictability.

\section{Acknowledgments}

The authors thank Marie-Eve Choronzey, Karolyn Forget, Marina Gilca, Marie-Claude Perron, and Élodie Samson for their technical and/or intellectual support.

Preliminary results presented at the annual meeting of the Association for Research in Vision and Ophthalmology, Fort Lauderdale, Florida, United States, May 7, 2012.

Supported by The Canadian Institutes for Health Research, Ottawa, ON, Canada and The FRQS Research in Vision Network, Montreal, QC, Canada. IB holds the Charles-Albert Poissant Research Chair in Corneal Transplantation, University of Montreal, Canada. Funding organizations had no role in the design or conduct of this research.

Disclosure: G.M. Durr, None; E. Auvinet, None; J. Ong, None; J. Meunier, None; I. Brunette, None

\section{References}

1. Fagerholm P, Lagali NS, Ong JA, et al. Stable corneal regeneration four years after implantation of a cell-free 
recombinant human collagen scaffold. Biomaterials. 2014;35: 2420-2427.

2. Zhang YQ, Zhang WJ, Liu W, et al. Tissue engineering of corneal stromal layer with dermal fibroblasts: phenotypic and functional switch of differentiated cells in cornea. Tissue Eng Part A. 2008;14:295-303.

3. Tan XW, Beuerman RW, Shi ZL, et al. In vivo evaluation of titanium oxide and hydroxyapatite as an artificial cornea skirt. J Mater Sci Mater Med. 2012;23:1063-1072.

4. Xiao X, Pan S, Liu X, et al. In vivo study of the biocompatibility of a novel compressed collagen hydrogel scaffold for artificial corneas. J Biomed Mater Res A. 2014;102:1782-1787.

5. Gatinel D. Topographie cornéenne. 1st ed. Paris: ElsevierMasson; 2011.

6. Weisenthal RW, Afshari NA, Bouchard CS, Colby KA, et al. Examination techniques for the external eye and cornea. In: External Disease and Cornea. San Francisco, CA: American Academy of Ophtalmology; 2013-2014:22-30.

7. Laliberté JF, Meunier J, Chagnon M, Kieffer JC, Brunette I. Construction of a 3-D atlas of corneal shape. Invest Ophthalmol Vis Sci. 2007;48:1072-1078.

8. Gatinel D, Haouat M, Hoang-Xuan T. A review of mathematical descriptors of corneal asphericity [in French]. J Fr Ophtalmol. 2002;25:81-90.

9. Fisher NI. Statistical Analysis of Circular Data. Cambridge, UK: Cambridge University Press; 1996:1-296.

10. Dubbelman M, Sicam VA, Van der Heijde GL. The shape of the anterior and posterior surface of the aging human cornea. Vision Res. 2006;46:993-1001.

11. Gatinel D, Malet J, Hoang-Xuan T, Azar DT. Corneal elevation topography: best fit sphere, elevation distance, asphericity, toricity, and clinical implications. Cornea. 2011;30:508-515.

12. Boote C, Hayes S, Abahussin M, Meek KM. Mapping collagen organization in the human cornea: left and right eyes are structurally distinct. Invest Ophthalmol Vis Sci. 2006;47:901908.

13. Smolek MK, Klyce SD, Sarver EJ. Inattention to nonsuperimposable midline symmetry causes wavefront analysis error. Arch Ophthalmol. 2002;120:439-447.

14. Wang L, Koch DD. Anterior corneal optical aberrations induced by laser in situ keratomileusis for hyperopia. $J$ Cataract Refract Surg. 2003;29:1702-1708.

15. Lombardo $\mathrm{M}$, Lombardo $\mathrm{G}$, Serrao $\mathrm{S}$. Interocular high-order corneal wavefront aberration symmetry. J Opt Soc Am A Opt Image Sci Vis. 2006;23:777-787.

16. Courville CB, Smolek MK, Klyce SD. Contribution of the ocular surface to visual optics. Exp Eye Res. 2004;78:417-425.

17. Bao FJ, Yu AY, Kassem W, Wang QM, Elsheikh A. Biometry of the cornea in myopic chinese patients. J Refract Surg. 2011; 27:345-355.

18. Dingeldein SA, Klyce SD. The topography of normal corneas. Arch Ophthalmol. 1989;107:512-518.

19. Falavarjani KG, Modarres M, Joshaghani M, Azadi P, Afshar AE, Hodjat P. Interocular differences of the Pentacam measurements in normal subjects. Clin Exp Optom. 2010;93:26-30.

20. Jonuscheit S, Doughty MJ. Repeatability of central corneal thickness measures by Orbscan pachymetry for right and left eyes. Eye Contact Lens. 2009;35:20-25.
21. McKendrick AM, Brennan NA. The axis of astigmatism in right and left eye pairs. Optom Vis Sci. 1997;74:668-675.

22. Myrowitz EH, Kouzis AC, O'Brien TP. High interocular corneal symmetry in average simulated keratometry, central corneal thickness, and posterior elevation. Optom Vis Sci. 2005;82: 428-431.

23. Dienes L, Kranitz K, Juhasz E, et al. Evaluation of intereye corneal asymmetry in patients with keratoconus. A Scheimpflug imaging study. PloS One. 2014;9:e108882.

24. Henriquez MA, Izquierdo L Jr, Mannis MJ. Intereye asymmetry detected by Scheimpflug imaging in subjects with normal corneas and keratoconus. Cornea. 2013;32:779-782.

25. Kompotiatis PSMK. A quantitative method for the analysis of bilateral corneal asymmetry in different corneal categories. $J$ Optom. 2009;2:173-181

26. Saad A, Guilbert E, Gatinel D. Corneal enantiomorphism in normal and keratoconic eyes. J Refract Surg. 2014;30:542547

27. Cairns G, McGhee CN. Orbscan computerized topography: attributes, applications, and limitations. I Cataract Refract Surg. 2005;31:205-220.

28. Grupcheva CN, Riley AF, Craig JP, Malik TY, McGhee CN. Analyzing small-incision cataract surgery by Orbscan II fourthdimensional pachymetry mapping. J Cataract Refract Surg. 2002;28:2153-2158.

29. Lattimore MR Jr, Kaupp S, Schallhorn S, Lewis R IV. Orbscan pachymetry: implications of a repeated measures and diurnal variation analysis. Ophthalmology. 1999;106:977-981.

30. Al-Torbak AA, Al-Motowa S, Al-Assiri A, et al. Deep anterior lamellar keratoplasty for keratoconus. Cornea. 2006;25:408412

31. Feizi S, Javadi MA, Jamali H, Mirbabaee F. Deep anterior lamellar keratoplasty in patients with keratoconus: big-bubble technique. Cornea. 2010;29:177-182.

32. Fournie P, Coullet J, Moalic S, Malecaze F, Chapotot E, Arne JL. Deep anterior lamellar keratoplasty in the surgical treatment of keratoconus. A 1-year follow-up [in French]. $J \mathrm{Fr}$ Ophtalmol. 2006;29:602-613.

33. Kubaloglu A, Koytak A, Sari ES, Akyol S, Kurnaz E, Ozerturk Y. Corneal endothelium after deep anterior lamellar keratoplasty and penetrating keratoplasty for keratoconus: a four-year comparative study. Indian J Ophthalmol. 2012;60:35-40.

34. Noble BA, Agrawal A, Collins C, Saldana M, Brogden PR, Zuberbuhler B. Deep anterior lamellar keratoplasty (DALK): visual outcome and complications for a heterogeneous group of corneal pathologies. Cornea. 2007;26:59-64.

35. Frost NA, Wu J, Lai TF, Coster DJ. A review of randomized controlled trials of penetrating keratoplasty techniques. Ophthalmology. 2006;113:942-949.

36. Troutman RC, Swinger CA, Belmont S. Selective positioning of the donor cornea in penetrating keratoplasty for keratoconus: postoperative astigmatism. Cornea. 1984;3:135-139.

37. Belmont SC, Troutman RC, Buzard KA. Control of astigmatism aided by intraoperative keratometry. Cornea. 1993;12:397400 .

38. Grütters G, Reichelt JA, Nolle B. Astigmatism-oriented perforating keratoplasty. A possibility for minimizing postoperative astigmatism [in German]? Ophthalmologe. 2001;98: 397-401 